Case Report

\title{
Unusual Lipomatous Lesion with Extramedullary Hematopoiesis Possibly Derived from Ito (Perisinusoidal) Cells in a CD-1 Mouse
}

\author{
Yuri Kotani ${ }^{1}$, Tetsuro Kurotaki ${ }^{1}$, Takuya Doi $^{1}$, Yumi Wako ${ }^{1}$, and Minoru Tsuchitani ${ }^{1}$ \\ ${ }^{1}$ Mitsubishi Chemical Safety Institute Ltd., 14 Sunayama, Kamisu-shi, Ibaraki-ken 314-0255, Japan
}

\begin{abstract}
A grayish white nodule, $1.0 \times 1.0 \times 2.0 \mathrm{~cm}$ in size, was grossly observed in the liver of a 110-week-old male CD-1 mouse. Microscopically, the lesion was characterized by two types of proliferation area, one of which was composed of a lipomatous proliferation with small spindle cells and severe extramedullary hematopoiesis (EMH). The other area was composed of large spindle cells closely proliferating along the hepatic cords. The proliferative lesion was partly positive for vimentin and had increased amounts of laminin, which is an extracellular matrix protein synthesized by Ito cells. Ultrastructurally, the lipomatous area contained cells with individual lipid droplets in the cytoplasm. The characteristic findings of this nodule were similar to those of Ito cell tumors in mice reported previously, except for the severe EMH in our case. (J Toxicol Pathol 2007; 20: 105-109)
\end{abstract}

Key words: CD-1 mice, extramedullary hematopoiesis, Ito cells, Ito cell tumor, liver tumor

Lipomatous lesions in the livers of mice were first mentioned as Ito cell hyperplasia or neoplasia in the National Toxicology Program (NTP) database ${ }^{1}$.

Subsequently, they were examined in detail using 13 lipomatous lesions in $\mathrm{B} 6 \mathrm{C} 3 \mathrm{~F} 1$ mice, but the origin of the proliferating cells could not be determined ${ }^{2}$. Recently, a report was published that referred to the origin of lipomatous lesions, in which 8 lipomatous lesions in the livers of CBA/J mice were detected immunohistochemically ${ }^{3}$. The authors suggested that the proliferating cells were of Ito cell origin because of reactivity to laminin and tenascin in the proliferating area. The present report describes a similar lesion in a CD-1 mouse, except for observation of extramedullary hematopoiesis.

The animal was a 110-week-old non-treated male CD-1 mouse used in a 2 year background data accumulation study for carcinogenicity studies submitted for scheduled sacrifice (105 weeks) for this purpose. It was cared for according to the principles outlined in the guide for the care and use of laboratory animals prepared by the Japanese Association for Laboratory Animal Science and our institution. At necropsy, a $1.0 \times 1.0 \times 2.0 \mathrm{~cm}$ grayish white nodule was identified in the papillary process of the caudate lobe of the liver. The tissue was fixed in $10 \%$ phosphate-buffered formalin

Received: 17 October 2006, Accepted: 18 April 2007

Mailing address: Yuri Kotani, Mitsubishi Chemical Safety Institute

Ltd., 14 Sunayama, Kamisu-shi, Ibaraki, 314-0255, Japan

TEL: 81-479-46-2871 FAX: 81-479-46-2874

E-mail: y-kotani@ankaken.co.jp solution and was subjected to routine histological examination using hematoxylin and eosin staining. Sections were additionally stained with reticulin silver impregnation and Masson's trichrome. Immunohistochemical staining for cytokeratin, vimentin, S-100, von Willebrand factor, $\alpha$ smooth muscle actin ( $\alpha$-SMA), desmin, laminin, and proliferating cell nuclear antigen (PCNA) was also performed. All antibodies were purchased from Dako Japan Co., Ltd. (Kyoto, Japan) except for laminin, which was purchased from Sigma-Aldrich Japan Co., Ltd. (Tokyo, Japan). Table 1 presents the conditions for immunohistochemical staining. The ABC method (Dako Japan Co., Ltd.) was used for cytokeratin, S-100, von Willebrand factor, and laminin. The Polymer-Immuno Complex method (Dako Japan Co., Ltd.) was used for vimentin, $\alpha$-SMA, desmin, and PCNA. For electron microscopical examination, the tissue was deparaffinized and hydrophilized from the paraffin embedded block, fixed in $1 \%$ osmium, routinely processed and embedded in Epon, and then prepared for examination.

Histologically, the lesion was composed of two types of proliferation area (Fig. 1, insert). One area consisted of lipomatous proliferation of vacuolated cells surrounded by small spindle cells displacing the hepatic cords (Fig. 1). Severe and diffuse erythrocytic and granulocytic extramedullary hematopoiesis (EMH) was observed in this area. The vacuolated cells contained single large vacuoles similar to mature fat cells. The surrounding spindle cells contained pale cytoplasm and oval or elliptic nuclei that were smaller than those of the hepatocytes (Fig. 2). Mitotic 

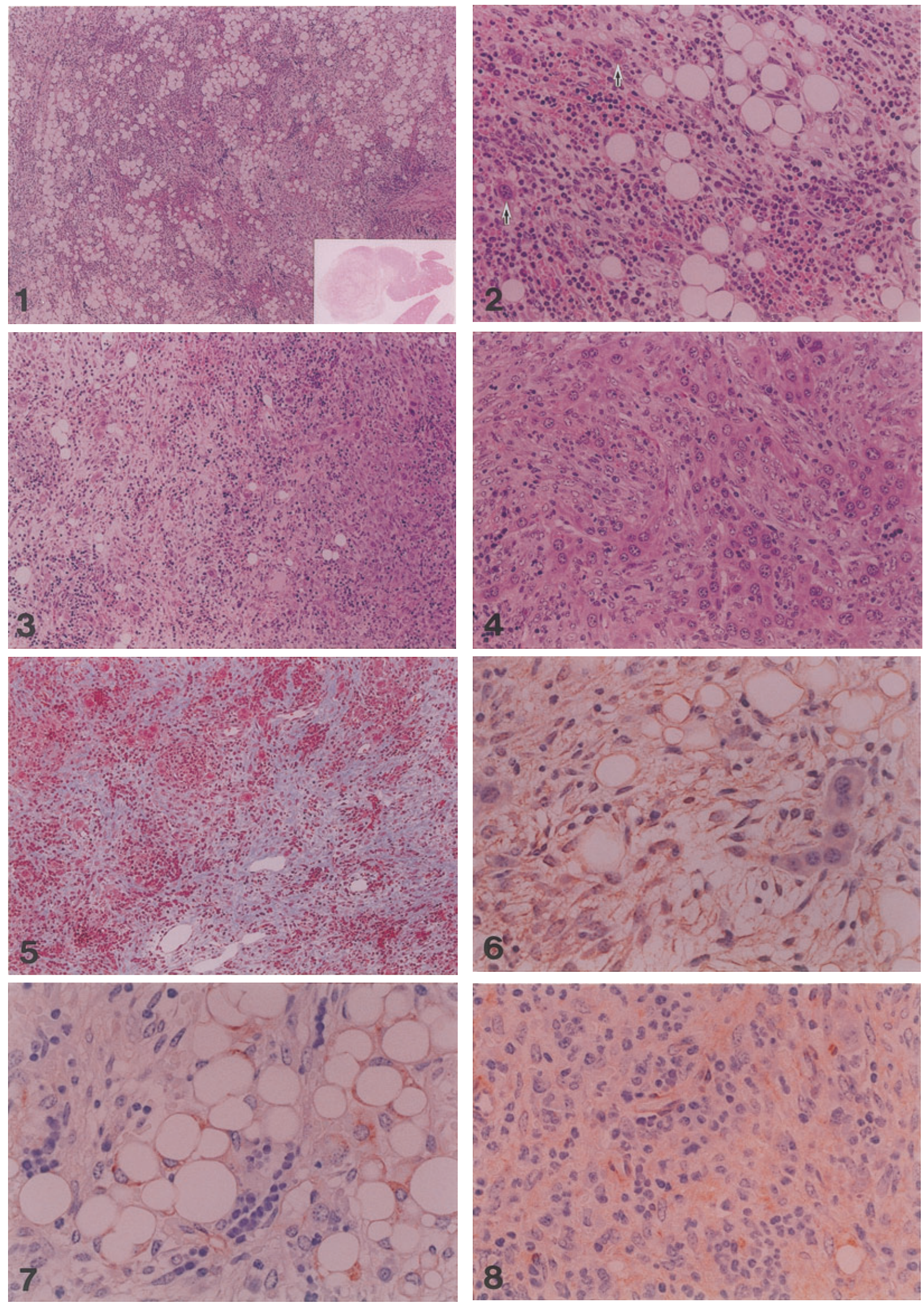
Table 1. Reagents for Immunohistochemistry

\begin{tabular}{|c|c|c|c|}
\hline Primary antibodies to (type) & Origin & Dilution & Pretreatment \\
\hline Cytokeratin (polyclonal) & Rabbit anti-cow & $1: 400$ & Proteinase $\mathrm{K}, 0.4 \mathrm{mg} / \mathrm{ml}, 5 \mathrm{~min}$ \\
\hline Vimentin (V9) & Mouse anti-porcine & $1: 50$ & Proteinase $\mathrm{K}, 0.4 \mathrm{mg} / \mathrm{ml}, 5 \mathrm{~min}$ \\
\hline S-100 (polyclonal) & Rabbit anti-cow & $1: 400$ & Proteinase $\mathrm{K}, 0.4 \mathrm{mg} / \mathrm{ml}, 5 \mathrm{~min}$ \\
\hline Von Willebrand Factor (polyclonal) & Rabbit anti-human & Ready to use & Proteinase $\mathrm{K}, 0.4 \mathrm{mg} / \mathrm{ml}, 5 \mathrm{~min}$ \\
\hline Alpha-smooth muscle actin (1A4) & Mouse anti-human & $1: 100$ & Heat $\left(95^{\circ} \mathrm{C}\right)$ with citrate buffer $(\mathrm{pH} 6.0)$ \\
\hline Desmin (D33) & Mouse anti-human & $1: 50$ & Heat $\left(95^{\circ} \mathrm{C}\right)$ with target retreaval solution $(\mathrm{pH} 9.0)$ \\
\hline Laminin (polyclonal) & Rabbit anti-mouse & $1: 50$ & Proteinase K, $0.4 \mathrm{mg} / \mathrm{ml}, 5 \mathrm{~min}$ \\
\hline PCNA & Mouse anti-human & $1: 100$ & Heat $\left(95^{\circ} \mathrm{C}\right)$ with distilled water \\
\hline
\end{tabular}

figures were rarely observed. Isolated individual hepatocytes or some hepatic cords were distributed throughout the nodule. The other type of proliferation, which is composed of larger spindle-shaped cells with a few vacuolated cells, was observed at the margin of the nodule (Fig. 3). This proliferation spread out from the nodule. The cells contained more abundant cytoplasm and were elongated along the hepatic cords (Fig. 4). Mitotic figure and nuclear atypia of the proliferating cells were rarely observed. An increased amount of extracellular fibrous matrix was observed between the proliferating cells. Mild EMH was also observed in this area. The margin of this proliferating area was irregular. No clear transitional pattern between the two types of spindle-shaped cell was detected. No reticulin fibers were detected in either of these proliferating areas. Both types of spindle-shaped cell were stained grayish blue by Masson's trichrome. The extracellular fibrous matrix was also stained blue (Fig. 5). EMH was not observed except in the proliferating area of the liver. Although mild erythrocytic EMH was detected in the spleen, hematological examination revealed no significant changes suggesting anemia.

Immunohistochemically, cytokeratin, S-100, von Willebrand factor, $\alpha$-SMA, and desmin produced no reaction in the proliferating vacuolated cells or either type of spindle-shaped cell. Vimentin was expressed weakly in some of the small spindle-shaped and vacuolated cells (Fig. 6). No positive labeling for vimentin was observed in the larger spindle-shaped cells. Laminin expression was detected in the peripheral cytoplasm of the vacuolated cells (Fig. 7). Furthermore, laminin expression was detected in the cytoplasm of some of the small spindle-shaped cells, which were also stained positive. The larger spindle-shaped cells and the extracellular matrix were barely positive for laminin (Fig. 8). The hepatocytes in both proliferating areas and the unaffected area did not express laminin. Very few proliferating cells were stained positive for PCNA.

Ultrastructurally, vacuolated cells contained a single large lipid droplet of medium density. In the close proliferative area, large spindle-shaped cells with poor cytoplasmic organelles were located between the hepatic cords, but a detailed location could not be defined (Fig. 9). Intracytoplasmic lysosomes and intermediate filaments were not detected in these cells. Extracellular fibrils were observed adjoining some of the spindle-shaped cells (Fig. 10). Spindle-shaped cells with some small lipid droplets in the cytoplasm were rarely observed (Fig. 11).

The lesions found in the liver of the CD-1 mouse consisted of lipid-laden (ultrastructurally confirmed) cells and small or large spindle-shaped cells with severe EMH. Observation of these two different types of proliferating cell, lipid-laden and spindle-shaped cells, is well in agreement with a previous published report concerning lipomatous proliferating lesions in mice ${ }^{2}$. However, the origin of the proliferating cells could not be defined in that report. Subsequently, similar lesions in the livers of $\mathrm{CBA} / \mathrm{J}$ mice were diagnosed as Ito cell tumors by immunohistochemical detection $^{3}$. Ito (perisinusoidal) cells are fat-storing cells in

Fig. 1. The pale area consists of lipomatous proliferation with small spindle-shaped cells displacing the normal hepatic tissue. Insert: the nodule occupying the papillary process of the caudate lobe. A pale area (left) can be observed. HE, $\times 100$.

Fig. 2. Vacuolated and small spindle-shaped cells with severe erythrocytic and granulacytic EMH. The nuclei of the proliferating cells are smaller than those of the hepatocytes (arrows). HE, $\times 200$.

Fig. 3. The margin of the pale area of Fig. 1. Insert: Close proliferation of large spindle-shaped cells (right) can be observed. HE, $\times 100$.

Fig. 4. The large spindle-shaped cells contain more abundant cytoplasm elongated along the hepatic cords. HE, $\times 200$.

Fig. 5. The extracellular matrix between the proliferating cells is stained blue by Masson's trichrome. Masson's trichrome, $\times 100$.

Fig. 6. Some of the small spindle-shaped and vacuolated cells are positive for vimentin. Polymer-Immuno Complex, hematoxylin counterstaining, $\times 400$.

Fig. 7. Vacuolated cells expressing laminin in their peripheral cytoplasm. ABC, hematoxylin counterstaining, $\times 400$.

Fig. 8. Laminin is expressed in the close proliferation area of larger spindle-shaped cells. ABC, hematoxylin counterstaining, $\times 400$. 


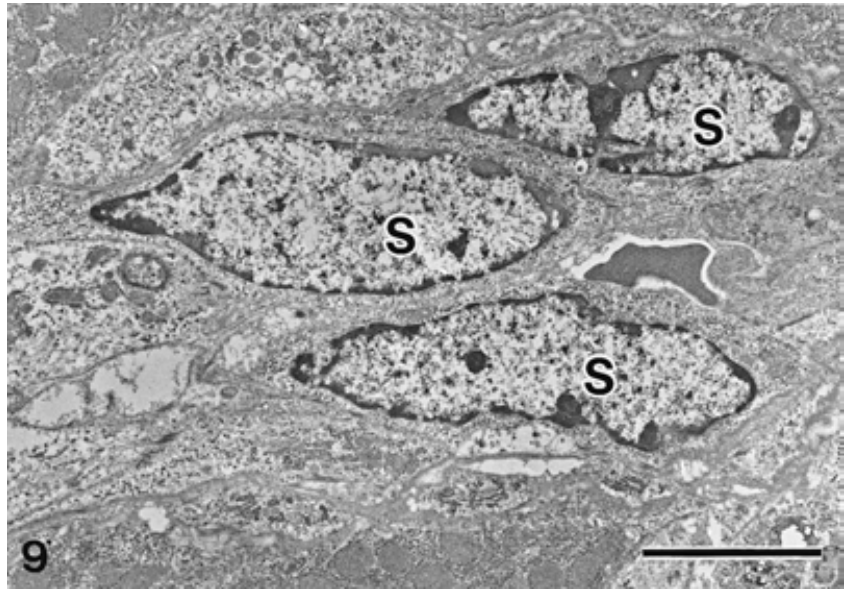

Fig. 9. Electron micrograph. Spindle-shaped cells (S) with poor cytoplasmic organelles proliferating between the hepatic cords. Bar $=5 \mu \mathrm{m}$.

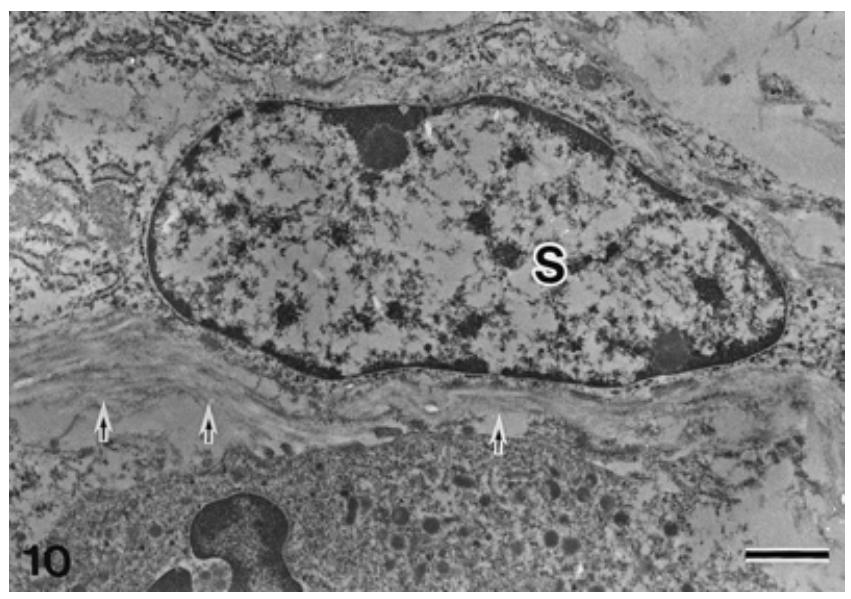

Fig. 10. Electron micrograph. Extracellular fibrils (arrows) adjoining spindle-shaped cells (S) can be observed. Bar $=1 \mu \mathrm{m}$.

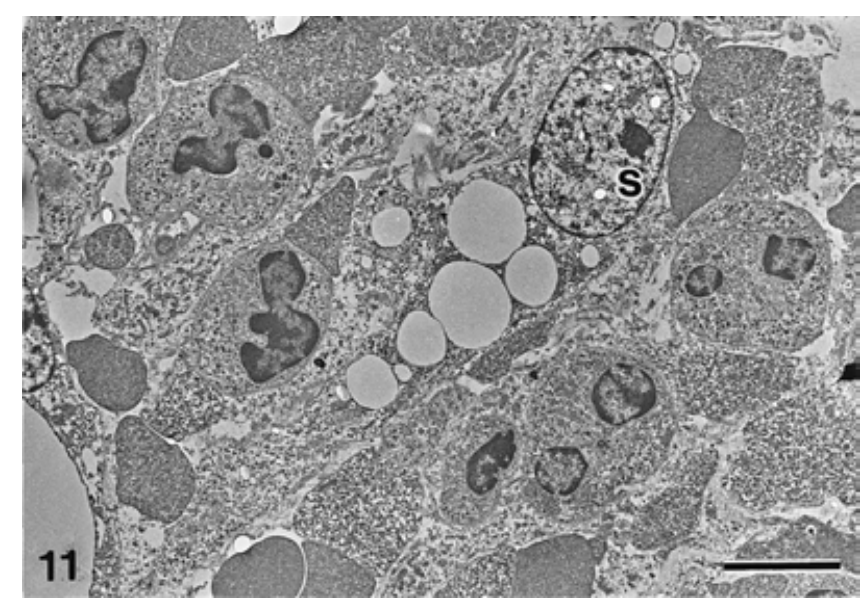

Fig. 11. Electron micrograph. Spindle-shaped cells (S) with small lipid droplets in the cytoplasm. Bar $=5 \mu \mathrm{m}$. the Disse's space that play a major role in the storage of Vitamin A and in the production of connective tissue and the basement membrane ${ }^{4}$. Lipomatous lesions in the liver are thought to be tumors originating from Ito cells ${ }^{1,5}$. In addition, neoplasms derived from Ito cells associated with spongiosis hepatis have been previously described in rats ${ }^{6,7}$. Although the mechanism remains unclear, the vacuolated (lipid-laden) and spindle-shaped cells in the present study could be phenotypes of Ito cells as reported previously in rats $^{7}$ and mice $^{3}$.

Immunohistochemical examination of the present case revealed that vimentin was partially expressed in some of the prolifelative cells. However, the cells did not react to desmin or $\alpha$-SMA. During proliferation, Ito cells in rats show phenotypic changes to myofibroblast-like cells (desminpositive/ $\alpha$-SMA-positive) and myofibroblast (desminnegative $/ \alpha$-SMA-positive $)^{8,9}$. This transformation may be influenced by various factors. In our case, proliferation was not accompanied by expression of these intermediate filaments. Laminin was expressed in the proliferating cells. Although laminin may not be a precise marker of Ito cells because of its expression in hepatocytes, ductal cells, and endothelial cells ${ }^{10}$, the proliferating cells in our case did not express cytokeratin or von Willebrand factor. Therefore, we suggest that these proliferating cells may be derived from Ito cells expressing and producing laminin. However, this study did not examine reactivity to tenascin produced exclusively by Ito cells. Detection of this protein is needed to confirm this diagnosis.

Ultrastructurally, a location characteristic of Ito cells was not detected. Proliferation of tumor cells into the Disse's space was not observed in previous cases of lipomatous lesions ${ }^{2}$. It would be difficult to define the origin of the lipomatous lesions in the liver based on ultrastructural location. As intracytoplasmic lysosomes were absent in the large spindle-shaped cells, they are unlikely to have originated from Kupffer cells. An extracellular fibrillar matrix adjoining these cells was observed in our case as described in a previous report of a human Ito cell tumor ${ }^{11}$. Spindle-shaped cells with multiple small lipid droplets in the cytoplasm may be transitional cells during transition to lipidladen cells from spindle-shaped cells, which may be the phenotypes of Ito cells.

Severe EMH was observed with proliferating cells in the present case. This occurs with proliferation of mature lipocytes such as myelolipoma, a kind of hamartomas, in adrenals, spleens, and livers. It is thought that myelolipoma/ myelolipomatous lesions in the liver may originate from Ito cells ${ }^{7}$ based on report of a human case ${ }^{12}$. However, the spindle-shaped cells in our case did not correspond to myelolipoma. It is more appropriate to diagnose this case as an Ito cell tumor with EMH than myelolipoma even if they have the same origin.

It remains unclear whether this lesion was benign or malignant. Very few proliferating cells were stained positive for PCNA. Moreover, there were almost no mitotic figures. The irregularity of the margin was similar to that in 
a previous report ${ }^{3}$. Additional animal cases are needed to define malignancy.

Although we could not confirm the diagnosis, the present lesion could be of Ito cell origin on the basis of its histological, immunohistochemical, and ultrastructural characteristics. To the best of our knowledge, this is the first report concerning an Ito cell tumor with EMH.

\section{References}

1. Maronpot RR, Haseman JK, Boorman GA, Eustis SE, Rao GN, and Huff JE. Liver lesions in B6C3F1 mice: The national toxicology program, experience and position. Arch Toxicol Suppl. 10: 10-26. 1987.

2. Dixon D, Yoshitomi K, Boorman GA, and Maronpot RR. "Lipomatous" lesions of unknown cellular origin in the liver of B6C3F1 mice. Vet Pathol. 31: 173-182. 1994.

3. Tillmann T, Kamino K, Dasenbrock C, Germann PG, Kohler M, Morawietz G, Campo E, Cardesa A, Tomatis L, and Mohr U. Ito cell tumor: Immunohistochemical investigations of a rare lesion in the liver of mice. Toxicol Pathol. 27: 364369. 1999.

4. Blomhoff R and Wake K. Perisinusoidal stellate cells of the liver: important roles in retinol metabolism and fibrosis. Fed Am Soc Exp Biol. 5: 271-277. 1991.

5. Eustis SL, Boorman GA, Harada T, and Popp JA. Liver.
Hyperplastic and neoplastic lesions. Miscellaneous lesions. Lipoma or liposarcoma. In: Pathology of the Fischer Rat, GA Boorman, SL Eustis, MR Elwell, CA Montgomery Jr, and WF Mackenzie (eds). Academic Press, San Diego, 8788. 1990.

6. Stroebel P, Mayer F, Zerban H, and Bannasch P. Spongiotic pericytoma: A benign neoplasm deriving from the perisinusoidal (Ito) cells in rat liver. Am J Path. 146: 903913. 1995.

7. Karbe E and Kerlin RL. Cystic degeneration/spongiosis hepatis in rats. Toxicol Pathol. 30: 216-227. 2002.

8. Hines JE, Johnson SJ, and Burt AD. In vivo response of macrophages and perisinusoidal cells to cholestatic liver injury. Am J Pathol. 142: 511-518. 1993.

9. Ogawa k, Suzuki J, Mukai H, and Mori M. Sequential changes of extracellular matrix and proliferation of Ito cells with enhanced expression of desmin and actin in focal hepatic injury. Am J Pathol. 125: 611-619. 1986.

10. Martinez-Hernandez A and Amenta PS. The extracellular matrix in hepatic regeneration. Fed Am Soc Exp Biol J. 9: 1401-1410. 1995.

11. Kaiserling E and Muller H. Neoplasm of hepatic stellate cells (spongiotic pericytoma): A new tumor entity in human liver. Pathol Res Pract. 201: 733-743. 2005.

12. Peters WM, Dixon MF, and Williams NS. Angiomyelolipoma of the liver. Histopathology. 7: 99-106. 1983. 